\title{
Application of the scanning ion-conductance microscopy (SICM) in study of voriconazole impact on Candida parapsilosis surface structure.
}

Nikita Savin ${ }^{1}$, Vasilii Kolmogorov², Roman Timoshenko ${ }^{3}$, Alexander Vaneev ${ }^{4}$, Aleksei Iakovlev ${ }^{2}, \mathrm{Oleg}^{2}$ Suchalko $^{2}$, Natalia Grammatikova ${ }^{5}$, Igor Levshin ${ }^{5}$, Natalia Klyachko ${ }^{6}$, Yurii Parkhomenko ${ }^{7}$, Sergei Salikhov ${ }^{7}$, Alexander Majouga ${ }^{8}$, Alexander Erofeev ${ }^{9}$, Peter Gorelkin ${ }^{9}$ and Yuri Korchev ${ }^{1}$

${ }^{1}$ National University of Science and Technology "MISIS", Moscow, Russia, ${ }^{2}$ National University of Science and Technology «MISiS», Moscow, Russia, Moskva, Russia, ${ }^{3}$ National University of Science and Technology "MISIS", Moscow, Moskva, Russia, ${ }^{4}$ National University of Science and Technology «MISiS», Russia, ${ }^{5}$ Gauze Research Institute for New Antibiotics, Moscow, Russian Federation, Russia, ${ }^{6}$ Lomonosov Moscow State University, Moscow, Russia, Russia, ${ }^{7}$ NUST MISiS, Moscow, Russian Federation, Russia, ${ }^{8} \mathrm{D}$. Mendeleev University of Chemical Technology, Russia, ${ }^{9}$ National University of Science and Technology «MISiS», Moscow, Russia, Russia

The studying of antifungal drugs effect on the surface structure by traditional microbiological methods is unobtainable. SICM method allows to obtain the topography of the surface structure and its mechanical properties of biological samples in non-contact mode (Clarke et al., 2016). Achievable to obtain a data of cells stiffness by applying pressure between of the nanopipette tip a sample surface. Azole drugs inhibit the synthesis of ergosterol (a component of the yeast membrane) (Ghannoum \& Rice, 1999), which lead to destruction and softening of candida cell membrane. There are reports of the antifungal effect of caspofungin on elasticity of cell wall (Quilès et al., 2017); impact of azole drugs on Candida surface structure (Madhavan et al., 2018; Behbehani et al., 2019). However, the dynamic effect of azole drugs on the surface structure and mechanical properties of Candida yeast in physiological conditions has not been studied. In this work, an impact of fluconazole on Candida parapsilosis. To perform an experiment, yeast immobilized on a glutaraldehyde layer was treated with voriconazole at $40 \mu \mathrm{g} / \mathrm{ml}$ for 6 hours. Cell morphology alteration of the Candida cell have been observed by SICM (figure 1). In addition, displacement map indicates increase at 2.5 times (figure 2 (A, B)). The average value of the displacement of the control cell was $17 \mathrm{~nm}$, the value after cell treatment was 41 $\mathrm{nm}$, which is indicated that the cell has softened after voriconazole treatment. Figure $2(\mathrm{C})$ presents a graph of the linear dependence of displacement value along the cell surface from the time of exposure with the drug. The curve of the graph does not reach a plateau due to the short exposure time of cells with drug (less than 24 hours). An increase in displacement value indicates a decrease in stiffness at the cell surface and, consequently, destruction of the Candida membrane. The data are consistent with theoretical concepts of the antifungal effect of the azole drugs on Candida yeast. However, this characteristic is indirect in determining the mechanical properties. Due to the relatively low pressure exerted on rigid yeast cells. Therefore, further refinement of the method for determining cell elasticity is relevant.

This work was supported by the Russian Science Foundation grant No. 19-19-00626. 


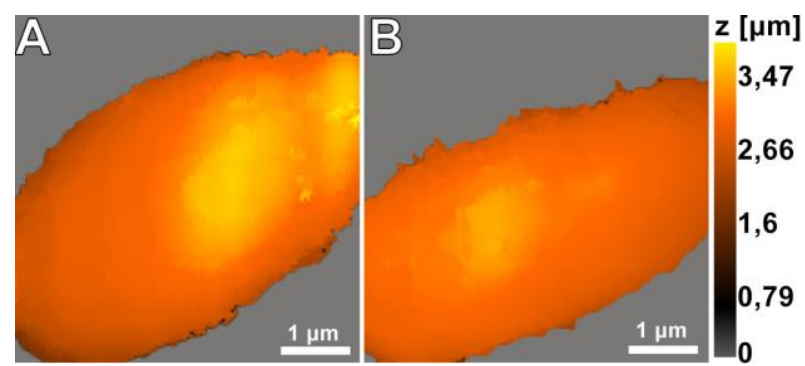

Figure 1. SICM images of Candida parapsilosis (A) control, (B) after 6 hours voriconazole treatment at 40 $\mu \mathrm{g} / \mathrm{ml}$.

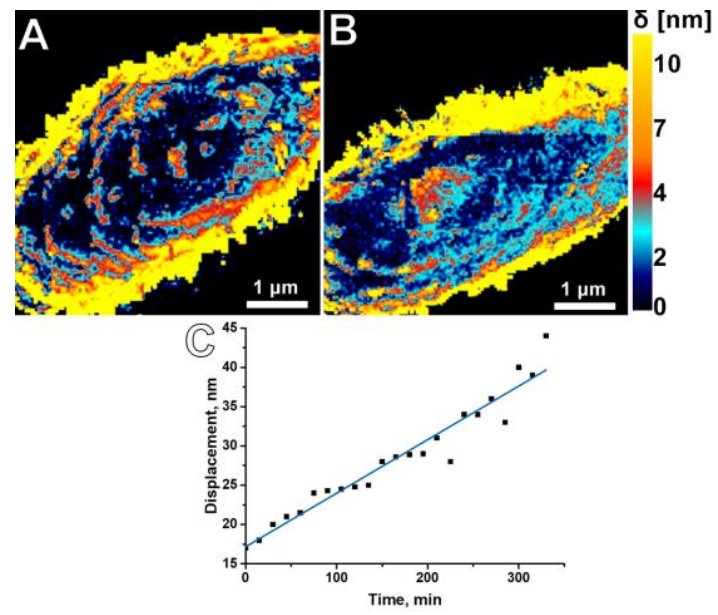

Figure 2. Surface displacement of Candida (A) control, (B) after 6 hours voriconazole treatment at $40 \mu \mathrm{g} / \mathrm{ml}$. (C) Time dependence of Candida surface displacement treated with voriconazole at $40 \mu \mathrm{g} / \mathrm{ml}$.

References

1. Clarke, R., Novak, P., Zhukov, A., Tyler, E., Cano-Jaimez, M., Drews, A., Richards, O., Volynski, K., Bishop, C. \& Klenerman, D. (2016). Low Stress Ion Conductance Microscopy of Sub-Cellular Stiffness. Soft Matter, 12 (38), 7953-7958.

2. Ghannoum, M. \& Rice, L. (1999). Antifungal agents: mode of action, mechanisms of resistance, and correlation of these mechanisms with bacterial resistance. Clin Microbiol Rev 12 (4), 501-517.

3. Quilès, F., Accoceberry, I., Couzigou, C., Francius, G., Noël, T. \& El-Kirat-Chatel, S. (2017). AFM combined to ATR-FTIR reveals Candida cell wall changes under caspofungin treatment. Nanoscale 9 (36), 13731-13738.

4. Madhavan, P., Jamal, F., Pei-Pei, C., Othman, F., Karunanidhi, A. \& Peng, K. (2018). Comparative Study of the Effects of Fluconazole and Voriconazole on Candida glabrata, Candida parapsilosis and Candida rugosa Biofilms. Mycopathologia 183, 499-511.

5. Behbehani, M., Irshad, M., Shreaz, S. \& Karche, M. (2019). Synergistic effects of tea polyphenol epigallocatechin 3-O-gallate and azole drugs against oral Candida isolates. Journal de Mycologie Médicale 29, 158-167. 